\title{
Clinicopathological and prognostic significance of epithelial mesenchymal transition-related protein expression in intrahepatic cholangiocarcinoma
}

This article was published in the following Dove Press journal:

OncoTargets and Therapy

II October 2012

Number of times this article has been viewed

\section{Xing Yao,* \\ Xiang Wangl,* \\ Zishu Wang ${ }^{2, *}$ \\ Licheng Dai' \\ Guolei Zhang' \\ Qiang Yan' \\ Weimin Zhou'}

'Huzhou Central Hospital, Zhejiang Huzhou, ${ }^{2}$ Department of Medical

Oncology, First Affiliated Hospital, Bengbu Medical College, Anhui,

People's Republic of China

*These authors contributed equally to this work
Correspondence: Xing Yao Huzhou Central Hospital, Zhejiang,

Huzhou 313000, China

Tel +86572202 330I

$\mathrm{Fax}+865722023301$

Email yaoxinghz@।63.com
Background: The aim of this study was to examine the patterns of expression of epithelialmesenchymal transition (EMT)-related proteins in intrahepatic cholangiocarcinoma. The clinicopathological and prognostic value of these markers was also evaluated.

Methods: We detected the expression status of three EMT-related proteins, ie, E-cadherin, vimentin, and N-cadherin, by immunohistochemistry in consecutive intrahepatic cholangiocarcinoma specimens from 96 patients

Results: The frequency of loss of the epithelial marker E-cadherin, and acquisition of mesenchymal markers, vimentin and N-cadherin, in intrahepatic cholangiocarcinoma was 43.8\%, 37.5\% and $57.3 \%$, respectively. Altered expression of EMT markers was associated with aggressive tumor behavior, including lymph node metastasis, undifferentiated-type histology, advanced tumor stage, venous invasion, and shorter overall survival. Moreover, loss of E-cadherin was retained as an independent prognostic factor for patients with intrahepatic cholangiocarcinoma in multivariate analysis.

Conclusion: Our results suggest that the EMT process is associated with tumor progression and a poor outcome in patients with intrahepatic cholangiocarcinoma, and inhibition of EMT might offer novel promising molecular targets for the treatment of affected patients.

Keywords: intrahepatic cholangiocarcinoma, epithelial-mesenchymal transition, expression, prognosis, immunohistochemistry

\section{Introduction}

Intrahepatic cholangiocarcinoma is the second most common primary cancer of the liver, and arises from malignant transformation of epithelial tissue in the small bile ducts. The prognosis of intrahepatic cholangiocarcinoma is very poor, with median survival for patients who do not undergo surgery being 6 months, and the 5-year survival rate for patients following complete resection being only $20 \%-40 \%{ }^{1,2}$ Being of different embryological origin, intrahepatic cholangiocarcinoma differs from extrahepatic cholangiocarcinoma and metastatic adenocarcinoma of the liver in both morphology and molecular background. ${ }^{3}$ In recent decades, the incidence of and mortality from intrahepatic cholangiocarcinoma has progressively increased, whilst being stable or slightly decreased for extrahepatic cholangiocarcinoma. ${ }^{4}$ Different stem cell niches have been identified recently in the liver and biliary tree, which might partly account for the difference between intrahepatic and extrahepatic cholangiocarcinoma. ${ }^{5}$ Specific novel therapeutic strategies are urgently needed for intrahepatic cholangiocarcinoma.

Epithelial-mesenchymal transition (EMT) is essential for phenotypic transition during embryogenesis and wound healing, and could also be reactivated during the 
malignant progression of numerous cancers. ${ }^{6}$ The process of EMT is characterized by loss of the epithelial marker, E-cadherin, abnormal nuclear location of $\beta$-catenin, and loss of acquired mesenchymal markers, such as vimentin and N-cadherin. Snail, sphingosine-1-phosphate, and Twist are considered to be master genes triggering EMT by strong repression of E-cadherin. ${ }^{7}$ Expression of EMT biomarkers has been investigated in various cancers, including those of gastric origin. ${ }^{8}$ However, the clinicopathological and prognostic significance of multiple EMT-related proteins in cholangiocarcinoma has not as yet been confirmed.

In this study, we investigated the expression of EMT markers in human intrahepatic cholangiocarcinoma, and their relationship with clinical features and prognosis was also determined.

\section{Materials and methods}

\section{Patients}

The study subjects included 96 patients with intrahepatic cholangiocarcinoma, who were surgically treated at Huzhou Central Hospital, Zhejiang, China, and the First Affiliated Hospital, Bengbu Medical College, Anhui, China, from 2002 to 2008. All tumors were confirmed pathologically. No patients underwent chemotherapy or radiotherapy before surgery. Postoperative adjuvant treatments were undertaken in a subset of patients with recurrence as follows: 12 cases received 5-fluorouracil-based chemotherapy; seven cases received radiotherapy, and five received both treatment modalities. Cases combined with extrahepatic cholangiocarcinoma were excluded. TNM (tumor-nodemetastasis) stages were based on pathological classification. Follow-up information was collected, including patient outcome and time interval between the date of surgical resection and cancer-related death. Patients lost to follow-up and those who died from causes other than cancer were considered as censored data. The median follow-up period was 23 (range 9-61) months. The study was approved by the institutional review board of our institutions, and informed consent was obtained from each patient.

\section{Immunohistochemistry}

Expression of EMT marker proteins, ie, E-cadherin, $\mathrm{N}$-cadherin, and vimentin and alpha-smooth muscle actin ( $\alpha$-SMA) was detected by immunohistochemistry. Briefly, $4 \mu \mathrm{m}$ thick sections were deparaffinized, rehydrated, and washed with phosphate-buffered saline. They were then treated with $3 \%$ hydrogen peroxide for 20 minutes at room temperature to inactivate endogenous peroxidases, followed by microwave antigen retrieval in $10 \mathrm{mM}$ citric acid ( $\mathrm{pH}$ 6.0) for 10 minutes. The slides were blocked with $3 \%$ bovine serum albumin in phosphate-buffered saline for 30 minutes and incubated with the mouse monoclonal antihuman E-cadherin antibody (Santa Cruz Biotechnology, Santa Cruz, CA, Clone G-10, \#sc-8426, at 1:100 dilution), mouse monoclonal antihuman vimentin antibody (Santa Cruz Biotechnology, Clone V9, \#sc-6260, at 1:100 dilution), mouse monoclonal antihuman vimentin antibody (Santa Cruz Biotechnology, Clone D-4, \#sc-8424, at 1:100 dilution), and mouse monoclonal antihuman $\alpha$-SMA antibody (Wuhan Boster Biological Technology, Fremont, CA, \# BM0002, at 1:100 dilution) overnight at $4^{\circ} \mathrm{C}$. Immunostaining was carried out using the Envision kit (DAKO, Carpinteria, CA) according to the manufacturer's instructions. Immunoreactivity was visualized with diaminobenzidine substrate and was then counterstained with hematoxylin. To create a positive tumor control marker, slides were incubated with rabbit anticytokeratin (DAKO) at 1:100. Mouse IgG at the same concentration instead of the primary antibodies was used as negative controls. Immunohistochemistry staining was evaluated according to a previous study of EMT markers. ${ }^{8}$ The sample was recorded in $200 \times$ fields, five different fields, and at least 1000 tumor cells were recorded in each sample. Cases were regarded as positive for each antibody when immunoreactivity was present in more than $10 \%$ of the cancer cells.

\section{Statistical analysis}

The Chi-square test was used to evaluate the statistical significance of the relationship between clinicopathological variables and expression of individual EMT-related proteins. The nonparametric Spearman rank correlation coefficient was applied to analyze for correlations between the expression of E-cadherin, vimentin, N-cadherin. The Kaplan-Meier method was used for univariate analysis of EMT markers to predict overall patient survival. A multivariate analysis of clinicopathological variables and EMT markers was performed using the Cox proportional hazard regression model. All statistical analyses were performed with SPSS version 16.0 software (SPSS Inc, Chicago, IL). Statistical significance was set at $P<0.05$.

\section{Results}

The clinical and pathological characteristics of the patients are shown in Table 1. Immunohistochemical staining was performed to analyze the protein expression of the epithelial protein marker, E-cadherin, and the mesenchymal markers, 


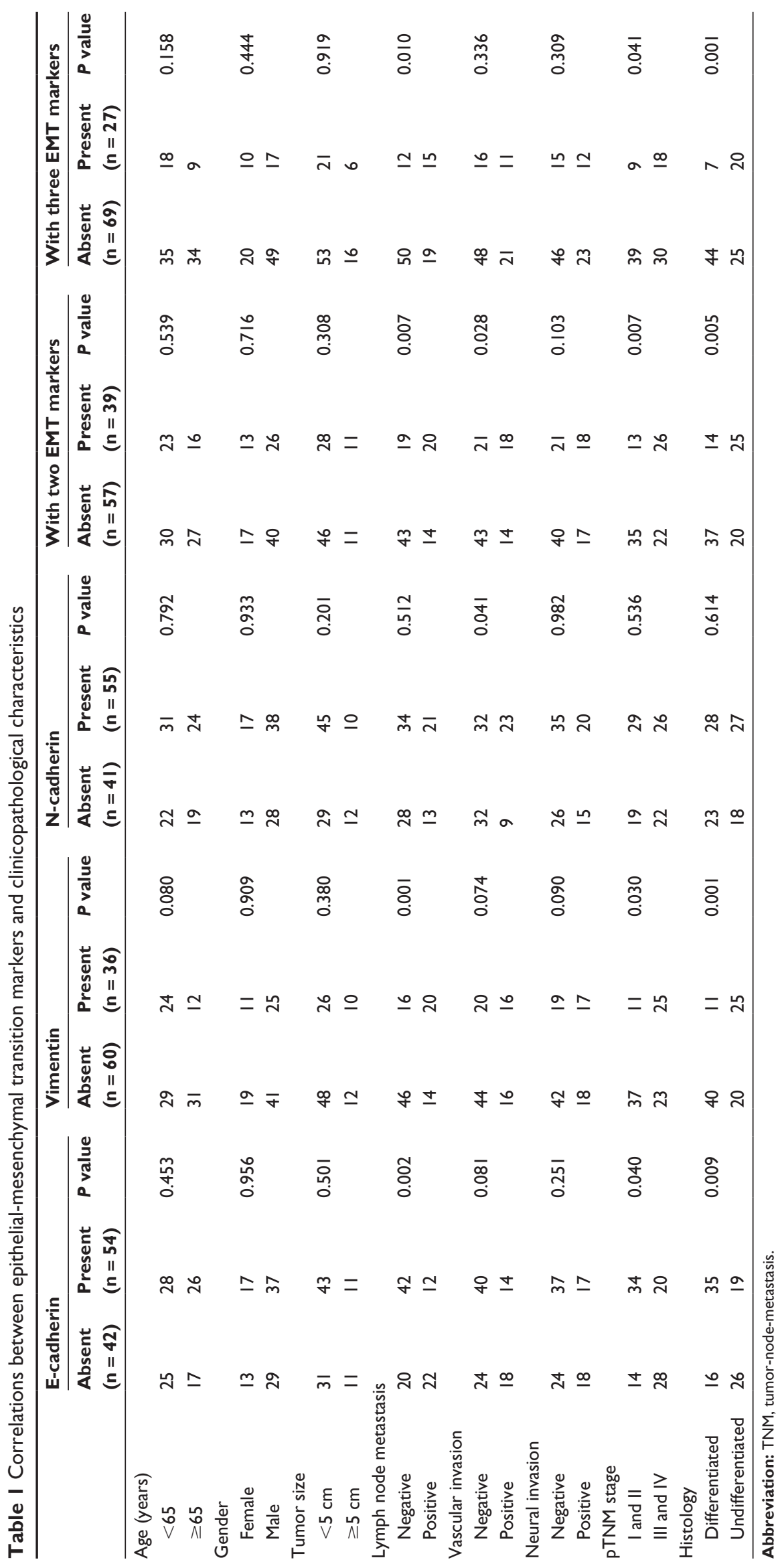


vimentin and $\mathrm{N}$-cadherin, in tumor specimens. Representative immunohistochemical results are shown in Figure 1. Epithelial E-cadherin protein loss frequency was observed in $43.8 \%$ (42/96) of tumors, and acquired mesenchymal vimentin and $\mathrm{N}$-cadherin protein overexpression frequency was seen in $37.5 \%(36 / 96)$ and $57.3 \%(55 / 96)$ of tumors, respectively.

The relationships between altered EMT protein expression and clinicopathological parameters are summarized in Table 1. Loss of E-cadherin and acquisition of vimentin were found to be significantly related to lymph node metastasis, advanced pTNM stage, and poorly differentiated type. In addition, N-cadherin-positive intrahepatic cholangiocarcinomas had a higher recurrence rate of vascular invasion $(41.8 \%, 23 / 55)$ than $\mathrm{N}$-cadherin negative tumors $(22.0 \%$, $P=0.041)$.

The Spearman rank correlation test showed that the three EMT-related proteins were closely related to each other in intrahepatic cholangiocarcinoma. Loss of E-cadherin was

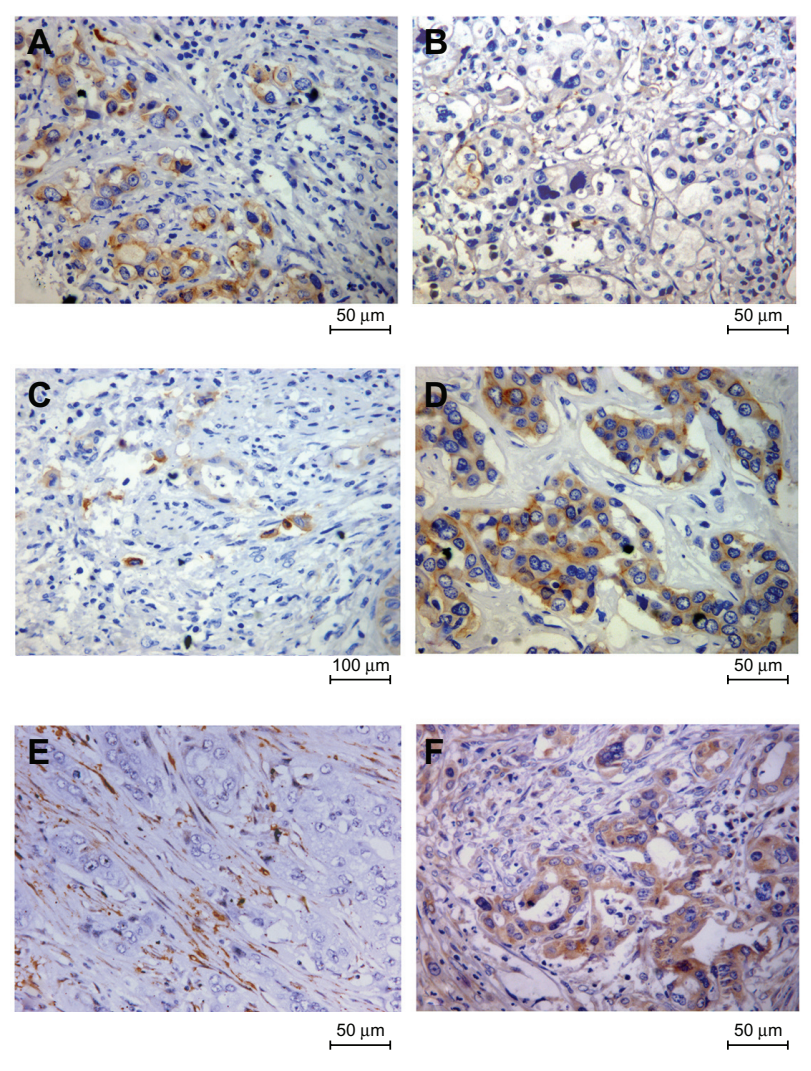

Figure I Representative immunohistochemical images of three epithelialmesenchymal transition markers in intrahepatic cholangiocarcinoma. (A) E-cadherin was strongly expressed in the plasma membrane of the tumor cells; (B) demonstration of loss of membranous $\mathrm{E}$-cadherin in tumor cells; (C) negative $\mathrm{N}$-cadherin expression with a positivity less than 10\%; (D) demonstration of $\mathrm{N}$-cadherin in the membrane and cytoplasm of tumor cells; (E) negative expression of vimentin in cancer cells while stromal cells were positively stained; and (F) acquired vimentin expression in cancer cells. negatively correlated with vimentin $(\mathrm{r}=-0.705, P=0.001)$ and N-cadherin $(\mathrm{r}=-0.422, P=0.001)$ expression. Membranous $\mathrm{N}$-cadherin expression also had a statistically significant correlation with increased vimentin expression ( $\mathrm{r}=0.277, P=0.006$ ). No significant relationship between stromal $\alpha$-SMA expression and EMT markers was observed in the intrahepatic cholangiocarcinoma tumors.

Overall survival was determined using the log-rank test with respect to expression of the three EMT markers (Figure 2). In terms of the epithelial marker, E-cadherin, loss was found to be significantly associated with a poor outcome. This was also true for upregulation of the mesenchymal proteins, vimentin and N-cadherin. The three EMT markers and four other statistically significant prognostic factors (lymph node metastasis, venous invasion, pTNM stage, and histology) identified in univariate analysis were
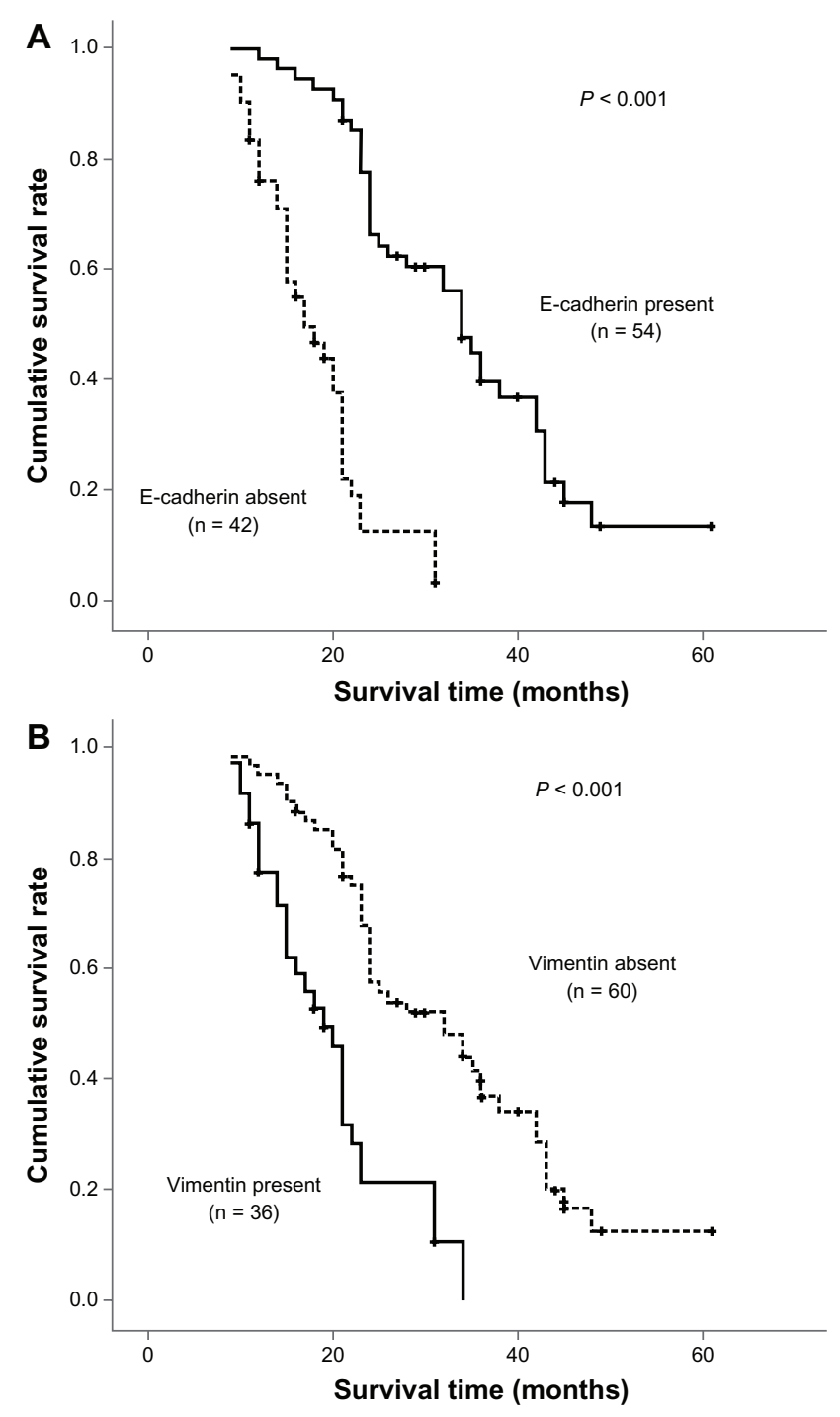

Figure 2 (Continued) 

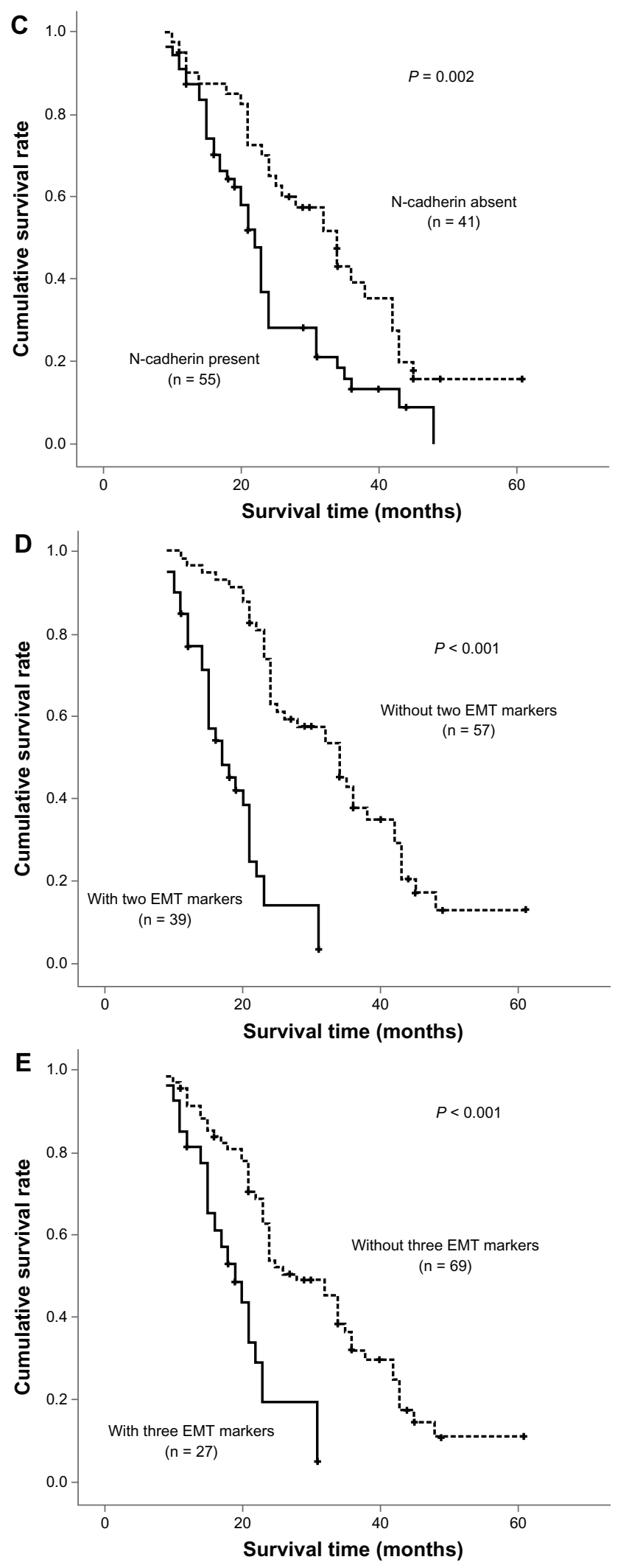

Figure 2 Survival analysis of three epithelial-mesenchymal transition markers in univariate analysis using the Kaplan-Meier method determined by the log-rank test. E-cadherin loss (A) and upregulation of mesenchymal proteins vimentin (B) and $\mathrm{N}$-cadherin (C) were found to be significantly associated with a poor outcome. There was also a trend toward coexistence of multiple epithelial-mesenchymal transition markers ( $\mathbf{D}$ and $\mathbf{E}$ ) associated with much more shorter overall survival. entered in the multivariate analysis. Among these factors, loss of E-cadherin expression and lymph node metastasis were shown to be independent prognostic indicators in patients with intrahepatic cholangiocarcinoma. However, vimentin and $\mathrm{N}$-cadherin expression had no prognostic significance in multivariate analysis (Table 2).

Among the positive cases, 39 tumors demonstrated coexistence of two EMT markers and 27 cases showed coexistence of three EMT markers. As seen in Table 1 and Figure 2, there was a trend towards coexistence of multiple EMT markers associated with lymph node metastasis, pTNM stage, histology, and shorter overall survival. Furthermore, there was also a significant association between coexistence of two EMT markers and vascular invasion.

\section{Discussion}

During progression of cancer, induction of EMT has been associated with acquisition of stemness properties, metastasis, and resistance to chemotherapy. ${ }^{9}$ The clinical and prognostic significance of EMT has been reported in various human cancers.

Although immunostaining of individual EMT markers, such as E-cadherin and $\beta$-catenin, has been reported in several previous studies involving small sample sizes, ${ }^{10,11}$ the expression profile of multiple EMT markers in intrahepatic cholangiocarcinoma has not been clarified.

Circumstantial evidence supports EMT being closely associated with progression of intrahepatic cholangiocarcinoma. Seol et a ${ }^{12}$ analyzed the gene profiling of nine cancer cell lines generated from intrahepatic

Table 2 Multivariate analysis of predictive factors for overall survival

\begin{tabular}{|c|c|c|c|}
\hline \multirow[t]{2}{*}{ Variables } & \multicolumn{3}{|l|}{ os } \\
\hline & HR & $95 \% \mathrm{Cl}$ & $P$ value \\
\hline \multicolumn{4}{|l|}{ LN metastasis } \\
\hline Positive versus negative & 2.416 & I.166-5.003 & 0.018 \\
\hline \multicolumn{4}{|l|}{ Vascular invasion } \\
\hline Positive versus negative & 1.222 & $0.703-2.124$ & 0.478 \\
\hline \multicolumn{4}{|l|}{ pTNM stage } \\
\hline I and II versus III and IV & 1.192 & $0.613-2.319$ & 0.604 \\
\hline \multicolumn{4}{|l|}{ Histology } \\
\hline Differentiated versus undifferentiated & 0.954 & $0.535-1.701$ & 0.873 \\
\hline \multicolumn{4}{|l|}{ E-cadherin } \\
\hline Present versus absent & 0.157 & $0.06 \mathrm{I}-0.402$ & 0.001 \\
\hline \multicolumn{4}{|l|}{ Vimentin } \\
\hline Present versus absent & 0.678 & $0.304-1.515$ & 0.344 \\
\hline \multicolumn{4}{|l|}{ N-cadherin } \\
\hline Present versus absent & 1.320 & $0.732-2.379$ & 0.356 \\
\hline
\end{tabular}

Abbreviations: HR, hazards ratio; LN, lymph node; TNM, tumor-node-metastasis; $\mathrm{OS}$, overall survival; $\mathrm{Cl}$, confidence interval. 
cholangiocarcinomas, and found upregulation of the EMTrelated proteins, vimentin and Twist1. Dos Santos et $\mathrm{al}^{13}$ examined the proteomic profiling of microdissected intrahepatic cholangiocarcinoma cells, and found that the protein alterations in these cells were very similar to those reported in the A549 human lung adenocarcinoma cell line during EMT induced by transforming growth factor beta, which has been demonstrated to be increased in intrahepatic cholangiocarcinoma. ${ }^{14,15}$ It has also been reported that reduced expression of beta-catenin is detected in a majority of biliary tumors, and their downregulation correlates with high-grade malignancy. ${ }^{16}$

In this study, we evaluated immunohistochemically the expression of three established EMT biomarkers which have been most frequently investigated in 96 consecutive intrahepatic cholangiocarcinoma specimens, and found that downregulation of E-cadherin and upregulation of vimentin and $\mathrm{N}$-cadherin was associated with aggressive biological behavior, including poor differentiation, advanced stage, positive lymph node involvement, and venous infiltration. Our findings are consistent with the findings of previous researchers. ${ }^{10,11}$

We extended the previous studies to confirm that expression of three EMT proteins was closely related in intrahepatic cholangiocarcinoma. Kaplan-Meier univariate analysis also showed that aberrant expression of the individual EMT-related proteins showed a trend toward poorer outcomes. Furthermore, loss of E-cadherin had an independent prognostic value in predicting outcomes in patients with primary intrahepatic cholangiocarcinoma. Taken together, our findings suggest that the EMT process may be an important molecular event during progression and metastasis of intrahepatic cholangiocarcinoma, which is in concordance with several published in vitro studies. Interaction of carcinoma and stromal cells plays an important role in the regulation of EMT in carcinomas. Schulte et $\mathrm{al}^{17}$ revealed significant correlations between stromal myofibroblasts and the EMT markers, Snail and Slug. However, in this study, using $\alpha$-SMA as a marker for stromal myofibroblasts, no statistically significant relationship was observed between stromal myofibroblasts and EMT markers in intrahepatic cholangiocarcinoma.

Given the aggressive nature and lack of effective adjuvant treatment modalities for intrahepatic cholangiocarcinoma, complete surgical resection is still the only therapeutic option for patients with the disease. However, even patients undergoing radical surgical resection are still at high risk for recurrence, and postoperative survival remains unsatisfactory.
Considering that the EMT phenotype is closely related to aggressive behavior and a poor prognosis in intrahepatic cholangiocarcinoma, we suggest that EMT might not only provide a new classification scheme, but might also offer a promising therapeutic target for patients with the disease. A couple of studies have proposed strategies to inhibit EMT. Li et al ${ }^{18}$ suggested that application of miR-214 might inhibit EMT by directly targeting the Twist gene in intrahepatic cholangiocarcinoma. Araki et $\mathrm{al}^{19}$ also indicated that transforming growth factor beta could be regarded as a candidate target to reverse EMT in biliary tumors.

\section{Conclusion}

In the present study, we have found that expression of EMTrelated proteins is closely associated with tumor progression and a poor prognosis in intrahepatic cholangiocarcinoma, suggesting that the EMT process may act as an important molecular event during the progression and metastasis of intrahepatic cholangiocarcinoma. We propose that EMT markers would have clinical implications for clinical classification; furthermore, inhibition of EMT might offer a novel promising target for the treatment of patients with intrahepatic cholangiocarcinoma.

\section{Disclosure}

The authors report no conflicts of interest in this work.

\section{References}

1. Yamamoto M, Ariizumi S. Surgical outcomes of intrahepatic cholangiocarcinoma. Surg Today. 2011;41:896-902.

2. Farges O, Fuks D. Clinical presentation and management of intrahepatic cholangiocarcinoma. Gastroenterol Clin Biol. 2010;34:191-199.

3. Sempoux C, Jibara G, Ward SC, et al. Intrahepatic cholangiocarcinoma: new insights in pathology. Semin Liver Dis. 2011;31:49-60.

4. Cardinale V, Semeraro R, Torrice A, et al. Intra-hepatic and extrahepatic cholangiocarcinoma: New insight into epidemiology and risk factors. World J Gastrointest Oncol. 2010;2:407-416.

5. Cardinale V, Carpino G, Reid L, Gaudio E, Alvaro D. Multiple cells of origin in cholangiocarcinoma underlie biological, epidemiological and clinical heterogeneity. World J Gastrointest Oncol. 2012;4:94-102.

6. Thiery JP. Epithelial-mesenchymal transitions in tumour progression. Nat Rev Cancer. 2002;2:442-454.

7. Thiery JP, Acloque H, Huang RY, Nieto MA. Epithelial-mesenchymal transitions in development and disease. Cell. 2009;139:871-890.

8. Kim MA, Lee HS, Lee HE, Kim JH, Yang HK, Kim WH. Prognostic importance of epithelial-mesenchymal transition-related protein expression in gastric carcinoma. Histopathology. 2009;54:442-451.

9. Mani SA, Guo W, Liao MJ, et al. The epithelial-mesenchymal transition generates cells with properties of stem cells. Cell. 2008;133:704-715.

10. Yu TH, Yuan RH, Chen YL, Yang WC, Hsu HC, Jeng YM. Viral hepatitis is associated with intrahepatic cholangiocarcinoma with cholangiolar differentiation and N-cadherin expression. Mod Pathol. 2011;24:810-819.

11. Zhang KJ, Zhang BY, Zhang KP, et al. Clinicopathologic significance of slug expression in human intrahepatic cholangiocarcinoma. World J Gastroenterol. 2010;16:2554-2547. 
12. Seol MA, Chu IS, Lee MJ, et al. Genome-wide expression patterns associated with oncogenesis and sarcomatous transdifferentation of cholangiocarcinoma. BMC Cancer. 2011;11:78.

13. Dos Santos A, Court M, Thiers V, et al. Identification of cellular targets in human intrahepatic cholangiocarcinoma using laser microdissection and accurate mass and time tag proteomics. Mol Cell Proteomics. 2010;9: 1991-2004.

14. Gal A, Sjoblom T, Fedorova L, Imreh S, Beug H, Moustakas A. Sustained TGF beta exposure suppresses Smad and non-Smad signalling in mammary epithelial cells, leading to EMT and inhibition of growth arrest and apoptosis. Oncogene. 2008;27:1218-1230.

15. Shimizu T, Yokomuro S, Mizuguchi Y, et al. Effect of transforming growth factor-betal on human intrahepatic cholangiocarcinoma cell growth. World J Gastroenterol. 2006;12:6316-6324.

16. AsayamaY, Taguchi Ki K, Aishima Si S, NishiH, Masuda K, Tsuneyoshi M. The mode of tumour progression in combined hepatocellular carcinoma and cholangiocarcinoma: an immunohistochemical analysis of E-cadherin, alpha-catenin and beta-catenin. Liver. 2002;22:43-50.
17. Schulte J, Weidig M, Balzer P, et al. Expression of the E-cadherin repressors Snail, Slug and Zeb1 in urothelial carcinoma of the urinary bladder: relation to stromal fibroblast activation and invasive behaviour of carcinoma cells. Histochem Cell Biol. 2012. [Epub ahead of print]

18. Li B, Han Q, Zhu Y, Yu Y, Wang J, Jiang X. Down-regulation of miR-214 contributes to intrahepatic cholangiocarcinoma metastasis by targeting Twist. FEBS J. 2012;279:2393-2398.

19. Araki K, Shimura T, Suzuki H, et al. E/N-cadherin switch mediates cancer progression via TGF-beta-induced epithelial-to-mesenchymal transition in extrahepatic cholangiocarcinoma. Br J Cancer. 2011;105: 1885-1893.

\section{Publish your work in this journal}

OncoTargets and Therapy is an international, peer-reviewed, open access journal focusing on the pathological basis of all cancers, potential targets for therapy and treatment protocols employed to improve the management of cancer patients. The journal also focuses on the impact of management programs and new therapeutic agents and protocols on

\section{Dovepress}

patient perspectives such as quality of life, adherence and satisfaction. The manuscript management system is completely online and includes a very quick and fair peer-review system, which is all easy to use. Visit http://www.dovepress.com/testimonials.php to read real quotes from published authors.

Submit your manuscript here: http://www.dovepress.com/oncotargets-and-therapy-journal 